\title{
Revision Hammer Toe Surgery
}

National Cancer Institute

\section{Source}

National Cancer Institute. Revision Hammer Toe Surgery. NCI Thesaurus. Code $C 96172$.

A therapeutic surgical procedure to reverse a hammer toe deformity. It involves bone removal and placement of special pins. 